Старков Н. В.

\title{
ВЛИЯНИЕ ФОРМЫ ШТАМПОВАННЫХ ЛИСТОВЫХ ДЕТАЛЕЙ НА ИХ ПРУЖИНЕНИЕ И УСТРАНЕНИЕ ЕГО ПРИ ИМПУЛЬСНОЙ ЭЛЕКТРОГИДРАВЛИЧЕСКОЙ КАЛИБРОВКЕ
}

Высокопрочные стали с пределом прочности 800-1100 МПа в последние годы стали предметом исследования как предприятий, их использующих, так и научных учреждений, которые изучают их свойства в технологических процессах листовой штамповки. Эти стали создавались для изготовления кузовов транспортных машин, к которым, наряду с высокой прочностью, предъявляются требования высокого уровня пластичности, технологичности при выполнении сборочных операций и др. требования.

Одной из особенностей этих сталей считается их значительное деформационное упрочнение и, как следствие, возникающее после штамповки деталей пружинение, которое часто превышает допустимые для каждого конкретного случая нормы $[1,2]$.

Пружинение - это изменение размеров штампованных деталей по сравнению с размерами, заданными инструментом, вследствие действия упругих напряжений после снятия деформирующей нагрузки, которые неравномерно распределены по толщине заготовки. При этом растянутые слои заготовки укорачиваются, а сжатые удлиняются. Разнонаправленность упругой деформации в зонах растяжения и сжатия вызывает поворот поперечного сечения штамповки на некоторый угол, называемый углом пружинения [3]. Пружинение может возникать также после штамповки тонких деталей облицовки кузова автомобиля, в которых неравномерность пластической деформации поверхности листа, например в результате создания на нём местной штамповки, может привести к появлению в детали значительной неравномерности напряжений и деформаций.

Целью данной работы является установить аналитическим путём связь формы штампуемых деталей с их пружинением и показать возможность импульсной электрогидравлической (ЭГ) калибровки для его устранения.

В статье рассматриваются детали, относящиеся к классу кузовных автомобильных деталей, которые после сборки автомобиля обеспечивают его необходимую прочность и жёсткость. Это, в основном, детали в виде длинного рифта, который в поперечном сечении имеет форму жёлоба. Такие детали отличаются значительным пружинением как в поперечном, так и в продольном направлениях, устранение которого представляет собой сложную технологическую задачу. Рассматривается пружинение деталей, изготовленных из высокопрочных листовых сталей путём вытяжки на механических прессах двойного действия в жестком штампе, который включает пуансон, матрицу и прижим.

Для прогнозирования пружинения детали после штамповки необходимо провести детальный анализ её конечной формы. Наиболее распространёнными в штамповке формами листовых деталей можно считать следующие:

- тела вращения с фланцем и без фланца;

- коробки квадратные и прямоугольные в плане с фланцем и без него;

- жёлоб прямолинейный и изогнутый открытый с двух сторон с фланцем и без него;

- детали открытого сложного профиля.

На рис. 1 приведены такие детали в аксонометрическом изображении. 


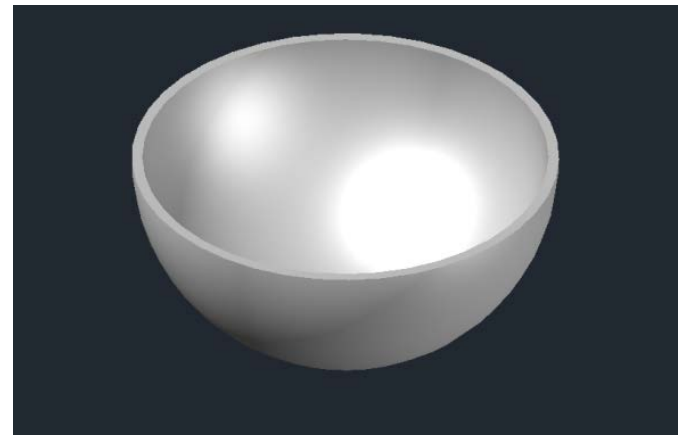

$\mathrm{a}$

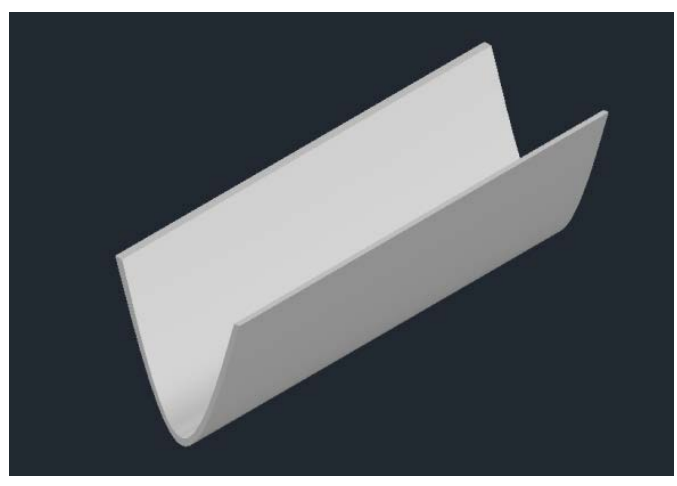

B

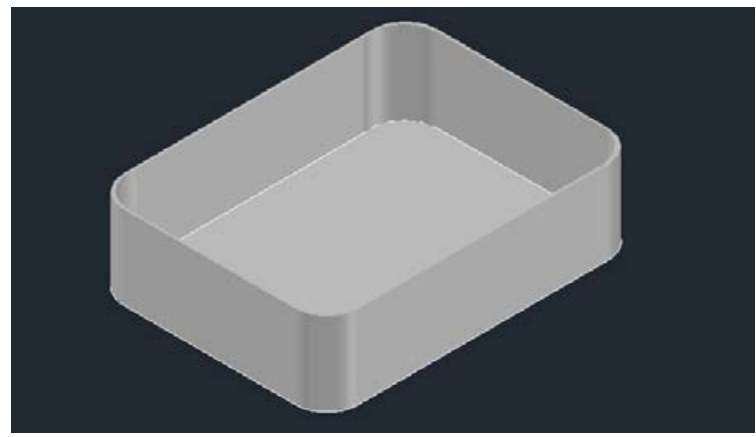

б

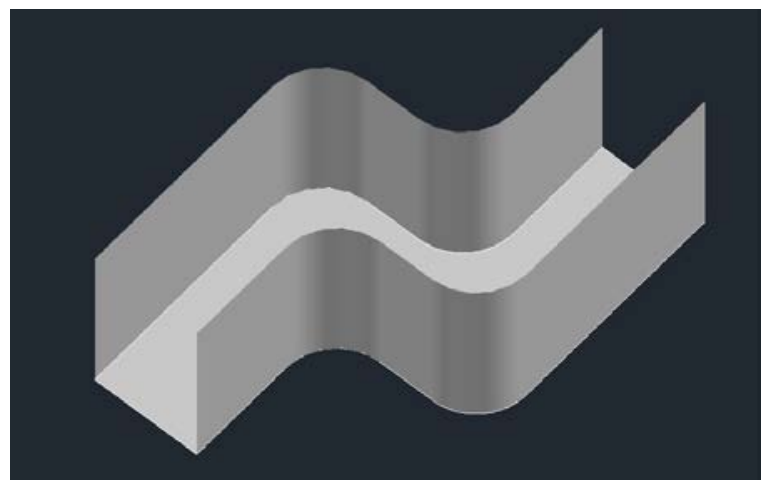

$\Gamma$

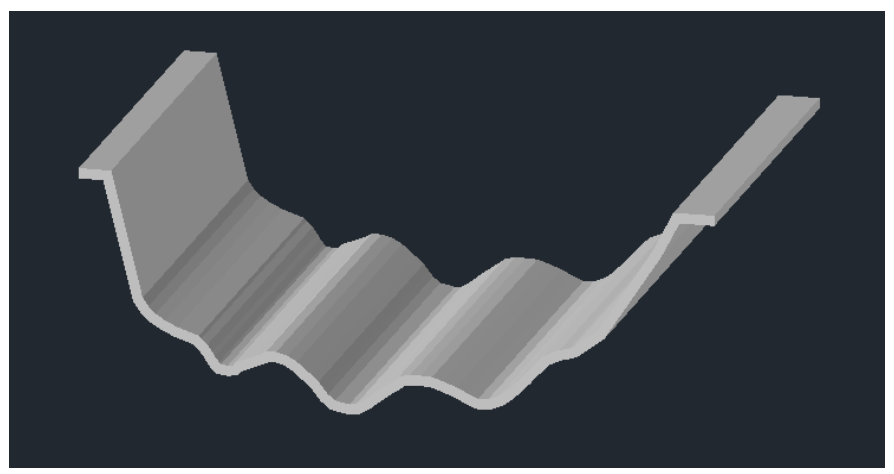

Д

Рис. 1. Форма листовых штампованных деталей для анализа степени их пружинения:

a - тело вращения; б - коробка; в - жёлоб прямолинейный; г - жёлоб изогнутый; д - открытый профиль

Пружинение таких деталей измеряют путем сравнения их линейных размеров в матрице и после извлечения её из матрицы. В операциях гибки для оценки пружинения часто пользуются угловыми измерениями отклонения формы стенок детали [4]. Такие измерения дают неполную информацию о величине пружинения, поскольку они будут одинаковыми у разных по размеру деталей, но чем меньше размеры деталей, тем труднее точно измерить это пружинение.

На рис. 2 показаны последовательные этапы формоизменения детали в процессе штамповки и анализируется процесс появления остаточных напряжений $(\mathrm{OH})$ в её стенках.

На начальной стадии процесса деформирования (а) заготовка под действием пуансона деформируется на 2-х сравнительно малых участках , длина которых с каждой стороны равна $L=\pi\left(R_{1}+R_{2}\right) / 2$. При этом изгибающие моменты на этих участках направлены в разные стороны. Если для плоского участка под пуансоном условия деформирования меняются мало, так как заготовка тут растягивается незначительно, то для участка изгиба на матрице они меняются непрерывно по мере движения пуансона вниз. Как только изогнутый участок выходит 
на вертикальную стенку он выравнивается (б) и тангенциальные напряжения на стенках детали уменьшаются. Если бы металл заготовки не упрочнялся, то после выравнивания заготовки на стенке матрицы напряжения сжатия и растяжения уменьшились бы до нуля и в стенках заготовки были бы только напряжения растяжения, которые создаёт перемещающийся пуансон. В действительности высокопрочные автомобильные стали отличаются большим деформационным упрочнением и поэтому после окончания вытяжки на стороне детали, обращённой к пуансону, напряжения сжатия исчезают или могут стать напряжениями растяжения, а на противоположной стороне они однозначно остаются растягивающими и большими, чем на другой стороне (в). После снятия нагрузки (остановка пуансона) и извлечения детали из штампа напряжения на обоих сторонах выравниваются, но для этого вертикальная стенка должна изогнуться в сторону больших растягивающих напряжений. В углах деталь под действием упругих сил, обусловленных напряжениями растяжения-сжатия, изгибается по простой схеме в сторону раскрытия прямого угла и после штамповки деталь приобретает форму, показанную на рис. 3. Эта деталь была изготовлена из высокопрочной стали СР 800 с пределом текучести $\sigma_{\text {т }}=686$ МПа и пределом прочности $\sigma_{\text {в }}=861$ МПа.

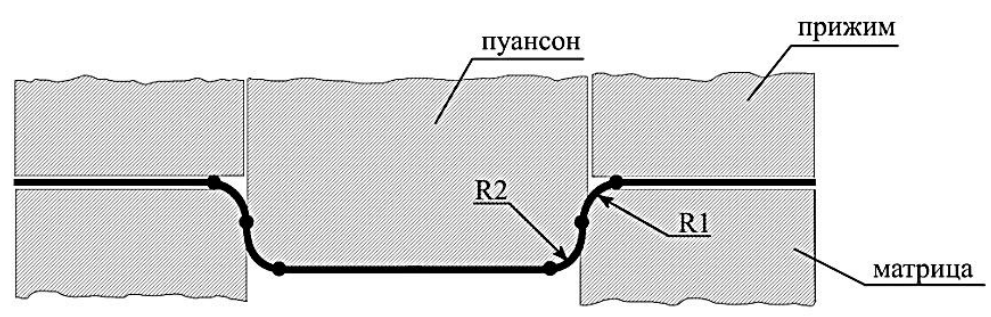

a

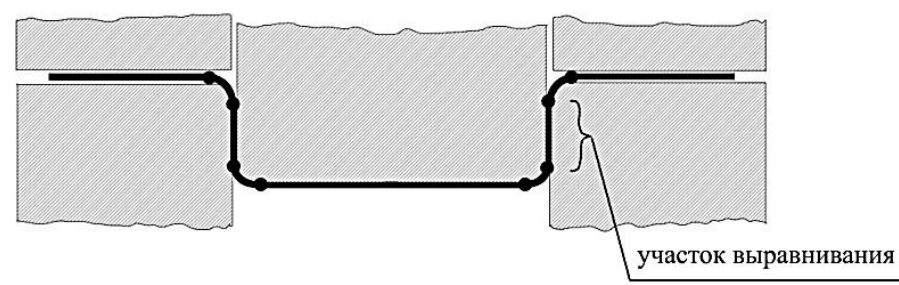

б

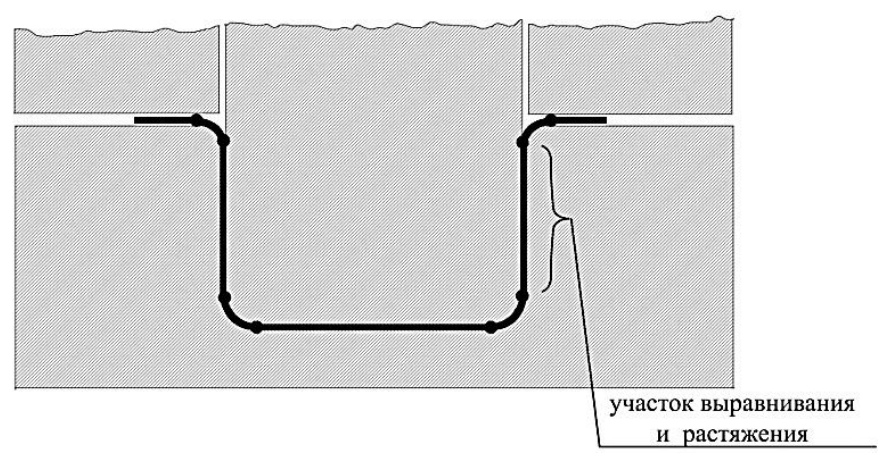

B

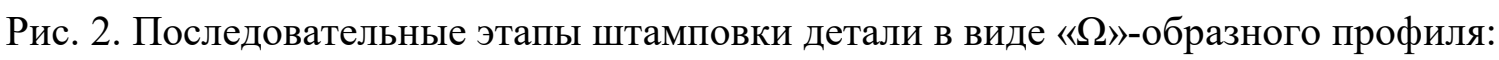
a - начальная стадия; б - выравнивание; в - выравнивание и растяжение

У некоторых деталей, изготовленных из высокопрочных сталей и отличающихся своей формой, пружинение может отсутствовать или быть очень незначительным. При этом имеется в виду, что под незначительным пружинением следует понимать отклонение размеров детали в пределах допуска заданного квалитета точности её изготовления. 
Так у деталей, изображённых на рис. 1, пружинение будет отсутствовать у деталей в форме тела вращения (а), коробчатой формы (б), не зависимо имеют ли они конструктивный фланец или нет. Это объясняется тем, что упругие линейные деформации в стенках детали с замкнутым контуром после штамповки не могут быть больше упругих окружных деформаций, величина которых $\varepsilon$ с учётом таких механических свойств материала, как предел текучести $\sigma$ т и нормальный модуль упругости, $E$ не превышает десятой доли процента $\left(\varepsilon=\sigma_{\mathrm{T}} / \mathrm{E}\right)$.

Если такие детали имеют конструктивный фланец, то из-за его жёсткости у деталей пружинение практически отсутствует и измерить его будет невозможно.

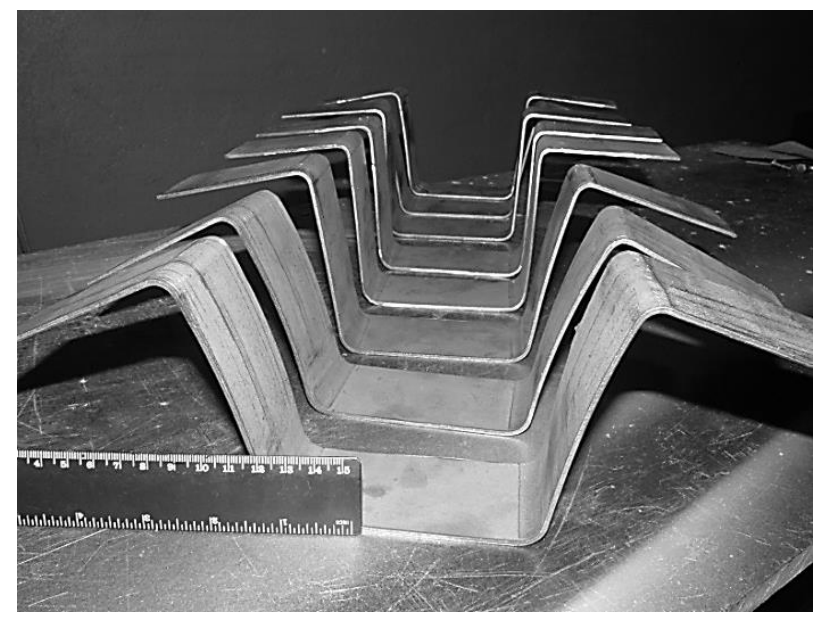

Рис. 3 Форма деталей после штамповки в вытяжном штампе с характерным видом пружинения

Совсем другой характер пружинения будет у штампованных деталей с открытым контуром в виде прямолинейного или изогнутого жёлоба (рис.1 в, г) с фланцем или без него; в виде открытого сложного профиля (рис. 1 д) и др. похожих деталей. В таких деталях пружинение возникает под действием $\mathrm{OH}$, механизм возникновения которых поясняется на рис. 2, а форма детали с характерным видом пружинения показана на рис. 3. Пружинение появляется как в поперечном сечении таких деталей, так и в продольном, если деталь имеет кривизну в этом направлении. Кроме такого пружинения, у деталей в форме изогнутого жёлоба (рис. 1 г) может появиться ещё и скручивающее пружинение.

Наиболее сложным представляет собой пружинение деталей сложной формы в виде открытого штампованного криволинейного профиля (рис. 1 д), у которых при штамповке появляются чередующиеся между собой криволинейные участки с растягивающими и сжимающими напряжениями. В таких деталях пружинение может быть устранено последовательной ЭГ калибровкой с двух сторон и применением, соответственно, двух матриц.

Устранение пружинения достигается различными способами. Термические способы, в виду специфики их применения, в статье не рассматриваются. Уменьшение пружинения при механической штамповке достигается корректировкой конструкции детали, и созданием таких условий процесса, при которых пружинение значительно снижается [3, 4]. Так в первом случае уменьшение пружинения в углах детали может быть достигнуто, если предусмотреть в ней элементы жесткости, которые штампуются вместе с деталью.

Во втором случае уменьшение углов пружинения достигается значительным уменьшением величины изгибающего момента, требующегося для деформирования. С уменьшением величины изгибающего момента, требуемого для заданного изменения кривизны, будет уменьшаться и угол пружинения, причем если изгибающий момент равен нулю, то все слои 
заготовки только растянуты или только сжаты до одинакового по величине напряжения; при разгрузке наблюдаются линейные деформации, а угловые (пружинение) отсутствуют.

Процесс формирования окончательных размеров детали качественно и пространственно неоднороден и протекает во времени с различной интенсивностью [5].

ЭГ калибровка с устранением пружинения имеет широкие возможности для эффективного применения. Устранение пружинения деталей при ЭГ калибровке достигается за счет релаксации остаточных напряжений в стенках детали. Волны напряжений и деформаций при прохождении в металле, воздействуя на кристаллическую решетку и ее дислокационную структуру, способствуют изменению его свойств. ЭГ калибровка ускоряет фазовые превращения, диффузионные и релаксационные процессы, способствует более равномерной деформации материала на микро- и субмикроуровнях, повышает сопротивление пластическому деформированию, увеличивает предельные остаточные деформации $[3,4,5]$.

Импульсное воздействие задается регулируемыми параметрами разрядного контура, определяющими энергию единичного разряда, а также числом разрядов при обработке изделия. В работе [6] показано, что эффективность релаксации ОН повышается с увеличением энергии единичного разряда как за счет увеличения зарядного напряжения, так и емкости батареи конденсаторов. Установлено, что увеличение числа разрядов способствует снижению ОН в изделии, однако эффективность импульсного воздействия от разряда к разряду убывает.

Уменьшение ОН пропорционально величине удельного импульса и зависит от его длительности, т. е. для уменьшения $\mathrm{OH}$ необходимо увеличивать удельный импульс и уменьшать его длительность [7]. Существует минимальный уровень удельного импульса, ниже которого уменьшение ОН не происходит, независимо от амплитуды волны давления при изменении постоянной времени экспоненциального уменьшения давления за фронтом волны от 10 до 40 мкс. Минимальное значение удельного импульса определяется уровнем ОН. Чем он ниже предела текучести, тем больше должна быть величина минимального удельного импульса..

Описанная кинетика процесса может быть объяснена тем, что по мере снижения ОН в результате перехода упругих деформаций в пластические в процессе накопления микроскопических сдвигов происходит как уменьшение напряжения, так и возрастание (за счет упрочнения) сопротивления пластическому деформированию.

Интенсификации процесса релаксации напряжений добиваются предварительным статическим нагружением деталей в области их упругих деформаций. При статической нагрузке и ЭГ калибровке изделий сумма остаточных и статических напряжений приближается к напряжениям предела текучести, что позволяет увеличить эффект релаксации напряжений. В статье [8] показано как в таких условиях в результате ЭГ обработки одним импульсом у группы деталей была исправлена и стабилизирована форма за счет устранения пружинения.

\section{ВЫВОДЫ}

Проведенный анализ связи формы листовых деталей штампуемых на механических прессах из высокопрочных сталей с их пружинением показал, что это свойство характерно для определённой группы деталей, форма которых соответствует определению «открытый жёлоб». Величина пружинения таких деталей может превысить размеры самих деталей и без исправления формы они не могут быть использованы.

Но есть класс деталей, пружинение которых отсутствует при любых режимах штамповки. В основном, это детали в виде тел вращения или коробок, имеющих замкнутый контур, и особенно тех, которые имеют конструктивный фланец. 
Процесс импульсной ЭГ калибровки деталей оказывается наиболее эффективным для устранения пружинения деталей после штамповки на механических прессах. При этом ЭГ калибровка деталей из высокопрочных сталей должна выполняться с предварительным созданием в них напряжений, близких к пределу текучести металла. Это позволяет более эффективно использовать процесс релаксации напряжений, при котором упругие деформации металла переходят в пластические.

\section{СПИСОК ИСПОЛЬЗОВАННОЙ ЛИТЕРАТУРЫ}

1. Advanced High-Strength SteelsApplication Guidelines, Version 5.0, May 2014.

2. Konieczny A. Advanced High Strength Steels - Formability. Great Designs in Steel Seminar, February 2003, American Iron and Steel Institute.

3. Попов Е. А. Основы теории листовой штамповки. Москва: Машиностроение, 1977. 280 с.

4. Романовский В. П. Справочник по холодной штамповке. Ленинград: Машиностроение, 1979. 520 с.

5. Головащенко С. Ф. Расширение технологических возможностей процессов листовой штамповки путем применения импульсных методов формообразования. ИПМСC: Maтериаль VIII Международной научной конференциии (17-21 августа 2009). Николаев, 2009. С. 49-51.

6. Тараненко М. Е. Метод снижения коробления листоштампованных деталей. Обработка материалов давлением: сборник научных трудов. Краматорск: ДГМА, 2018. № 1(46). С. 100-104.

7. Мазуровский Б. Я., Сизёв А. Н. Электрогидравлический эффект в листовой штамповке. Киев: Наукова думка, 1983. 192 с.

8. Старков Н. В., Стрелковская Л. И. Экспериментальное исследование пластичности алюминиевого сплава 2024-Т4 при импульсной электрогидравлической штамповке. Обработка материалов давлением: сборник научных трудов. Краматорск: ДГМА, 2019. № 1 (48). С. 82-87.

\section{REFERENCES}

1. Advanced High-Strength SteelsApplication Guidelines, Version 5.0, May 2014.

2. Konieczny A. Advanced High Strength Steels - Formability. Great Designs in Steel Seminar, February 2003, American Iron and Steel Institute.

3. Popov E. A. Fundamentals of the theory of sheet stamping. Moscow: Mechanical Engineering, 1977. 280p.

4. Romanovsky V.P. Reference book on cold stamping. Leningrad: Mechanical Engineering, 1979. 520 p.

5. Golovashchenko S. F. Expansion of technological capabilities of sheet stamping processes by applying impulse forming methods. IPMSS: Proceedings of the VIII International Scientific Conference (17-21 August 2009). Nikolaev, 2009. P. 49-51.

6. Taranenko M.E. Method of reducing warpage of stamped parts. Materials working by pressure. Kramatorsk: DSEA. 2018, No. 1 (46), pp. 100-104.

7. Mazurovsky, B. Ya. Electrohydraulic effect in sheet stamping. Kyiv: Naukova Dumka, 1983. 192 p.

8. Starkov N.V., Strelkovskaya L.I. Experimental study of the plasticity of aluminum alloy 2024-T4 during pulsed electrohydraulic stamping. Materials working by pressure. Kramatorsk: DSEA. 2019, No. 1 (48), pp. 82-87.

Старков Николай Владимирович - научный сотрудник ИИПТ.

ИИПТ - Институт импульсных процессов и технологий НАН Украины, г. Николаев.

E-mail: Starkovnikolay@yahoo.com 